\title{
Efeito da densidade de ninfas de Aphis gossypii Glover, 1877 (Hemiptera, Aphididae) no consumo alimentar e aspectos biológicos de Orius insidiosus (Say, 1832) (Hemiptera, Anthocoridae)
}

\author{
Simone M. Mendes ${ }^{1}$ \\ Vanda H.P. Bueno ${ }^{1}$ \\ Lívia M. Carvalho ${ }^{1}$ \\ Luís Cláudio P. Silveira ${ }^{1}$
}

\begin{abstract}
Effect of ninfal density of Aphis gossypii Glover, 1877 (Hemiptera, Aphididae) on feed consumption and biological aspects of Orius insidiosus (Say, 1832) (Hemiptera, Anthocoridae). The influence of different densities of $A$. gossypii $(10,20,30,40,50,60$ nymphs/day) on consumption rate, development time, survival, and reproduction of Orius insidiosus (Say, 1832) was investigated. The trials were carried out in climatic chamber at $25 \pm 1{ }^{\circ} \mathrm{C}, 70 \pm 10 \% \mathrm{RH}$, and photoperiod 12:12h (L:D). Consumption rates of nymphs and adults increased under a linear form as the densities of aphids increased. Development time was longest when reared in 10 nymphs density (15.4 days). Nymphal survival was different under the densities of A. gossypii and no significant difference for the periods of preoviposition (4.8 days) and oviposition ( 8.9 days) were found. The oviposition increased with the prey densities $(2.00,11.33,10.67,21.30,17.89$ and 53.38 eggs), as well as the viability: $0.00,52.49,57.86,58.14,50.11$ and $72.89 \%$, respectively. Nymphs of $A$. gossypii as prey were suitable for the complete development of $O$. insidiosus.
\end{abstract}

KEYwORDs. Biological control; development time; generalist predator; prey; reproduction.

\section{INTRODUÇÃO}

Orius insidiosus (Say, 1832) (Hemiptera, Anthocoridae) é um percevejo predador de pequenos artrópodes que vem sendo utilizado grandemente para o controle de tripes em cultivos protegidos na Europa (MEIRACKER 1999).

Vários autores (MALAis \& RAVESBERg 1992; VAN SCHELT 1993; MEIRACKER 1999) relatam a utilização de $O$. insidiosus para o controle de Frankliniella occidentalis (Pergande, 1895) (Thysanoptera, Thripidae) nos cultivos de pimentão (Capsicum annuum L.), pepino (Solanum murcatum L.) e crisântemo (Dendranthema grandiflora Tzelev). O pulgão Aphis gossypii Glover, 1877 (Hemiptera, Anthocoridae) é também uma praga nessas culturas, sugando a seiva das regiões meristemáticas, causando deformações nos brotos, além de ser responsável pela transmissão de viroses a essas espécies de plantas (BRiOso 1996; IMENES \& ALEXANDRE 1996).
Populações de predadores são capazes de persistir em habitats mais complexos devido à maior disponibilidade de recursos alimentares, sendo que a presença de presas alternativas pode ser fator determinante na manutenção do predador em um cultivo (Letourneau \& Altieri 1983) e, pelo fato de não ser dependente de uma espécie de presa, pode permanecer no ambiente previnindo infestações da presa-alvo. Entretanto, existem poucas referências sobre as interações do predador $O$. insidiosus com presas alternativas nos cultivos em que são empregados para o controle de tripes. Pulgões, bem como outras presas, têm sido observados sendo predados por espécies de Orius em cultivos protegidos. Segundo AlBAJES \& Alomar (1999), não há evidência da preferência de busca por nenhuma espécie com abundância de presas no campo. Assim, com o intuito de fundamentar o controle biológico de tripes e pulgões nas culturas onde estas pragas ocorrem em conjunto, este trabalho teve por objetivo verificar o efeito de

\footnotetext{
1. Departamento de Entomologia, Universidade Federal de Lavras. Caixa Postal 37, 37200-000 Lavras-MG, Brasil. Endereço eletrônico: smmendes@ufla.br.
} 
diferentes densidades de ninfas de $A$. gossypii no consumo alimentar e em outros aspectos biológicos do predador $O$. insidiosus.

\section{MATERIALE MÉTODOS}

O experimento foi desenvolvido no Departamento de Entomologia da Universidade Federal de Lavras, em câmaras climatizadas a $25 \pm 1{ }^{\circ} \mathrm{C}, 70 \pm 10 \%$ UR e $12 \mathrm{~h}$ de fotofase.

Uma criação do predador $O$. insidiosus foi mantida em laboratório de acordo com metodologia proposta por MENDES \& Bueno (2001). A presa A. gossypii foi criada em plantas de algodão, Gossypium hirsutum L. (Malvaceae), variedade Delta Pine, em sala climatizada com temperatura de $25 \pm 3{ }^{\circ} \mathrm{Ce} 12 \mathrm{~h}$ de fotofase.

Fase ninfal. $\mathrm{O}$ estudo foi conduzido com as densidades de $10,20,30,40,50$ e 60 ninfas do $1^{\circ}, 2^{\circ}$ e $3^{\circ}$ ínstares de $A$. gossypii, as quais foram oferecidas conjuntamente em cada densidade ao predador, em placas de Petri ( $5 \mathrm{~cm}$ de diâmetro) contendo um pequeno chumaço de algodão umedecido com água destilada e uma folha de algodoeiro (média de $14 \mathrm{~cm}^{2}$ ) que serviu como substrato de alimentação para as ninfas do pulgão. Ninfas recém eclodidas de $O$. insidiosus foram individualizadas nesses recipientes e diariamente as quantidades de ninfas do pulgão, em cada densidade estudada, foram oferecidas ao predador. As placas de petri foram vedadas com filme de PVC laminado e observadas diariamente sob microscópio estereoscópico quanto ao número de presas consumidas e à mudança de ínstar, nas diferentes densidades de presas. Foram considerados pulgões consumidos, aqueles em que o conteúdo do corpo foi removido parcial ou totalmente, de acordo com AlvarAdo et al. (1997). Também foi verificada a viabilidade da fase ninfal do predador frente às diferentes densidades oferecidas.

Fase adulta. Adultos de $O$. insidiosus foram alimentados com as diferentes densidades de ninfas de $A$. gossypii estudadas, em recipientes semelhantes aos utilizados para a fase ninfal do predador, sendo adicionada uma inflorescência de Bidens pilosa L. (Asteraceae) (previamente colocada em solução de hipoclorito de sódio $0,5 \%$, por 20 minutos para desinfecção da mesma) como substrato de oviposição. Os machos foram mantidos com as fêmeas até o início da postura. Diariamente, foram observados o consumo de presas, períodos de pré-oviposição e oviposição, número de ovos, número de fêmeas que ovipositaram e a longevidade dos adultos.

As inflorescências de $B$. pilosa contendo ovos de $O$. insidiosus foram individualizadas e envolvidas por um pequeno pedaço de algodão umedecido com água destilada e colocadas em placas de Petri $(5 \mathrm{~cm})$ vedadas com filme de PVC laminado. Estas foram observadas diariamente para avaliação da viabilidade dos ovos.

Análise estatística. O experimento foi realizado em delineamento inteiramente casualizado, composto por 6 tratamentos (10, 20, 30, 40, 50 e 60 ninfas $A$. gossypii). Os dados relativos ao período de desenvolvimento ninfal nos diferentes tratamentos foram submetidos à análise de variância. Foi realizada uma média diária do consumo de presas dentro de cada ínstar em cada densidade, e essas submetidas à análise de variância. Quando significativa, foi realizada uma análise de regressão. Quanto à viabilidade ninfal do predador, foi obtida a porcentagem de ninfas que sobreviveram em cada densidade estudada.

Para a fase adulta, foi feita a análise de variância para consumo de presas, períodos de pré-oviposição e oviposição, capacidade de oviposição das fêmeas e longevidade e, quando significativa, as médias foram submetidas ao teste de Sсотт \& KNOTT (1974), bem como à análise de regressão para o número de ovos /fêmea.

\section{RESULTADOSEDISCUSSÃO}

Fase ninfal. O período de desenvolvimento do $1^{\circ}$ ao $5^{\circ}$ ínstares de $O$. insidiosus variou nas diferentes densidades de A. gossypii testadas (Tabela 1), sendo que a menor densidade

Tabela 1. Período desenvolvimento $\left( \pm\right.$ EP) e viabilidade da fase ninfal de Orius insidiosus em função das diferentes densidades de presa, $25 \pm 1{ }^{\circ} \mathrm{C}$, $70 \pm 10 \%$ UR e $12 \mathrm{~h}$ de fotofase.

\begin{tabular}{cccc}
\hline \multirow{2}{*}{ Densidades } & N & $\begin{array}{c}\text { Período desenvolvimento } \\
\text { da fase ninfal }\end{array}$ & \multicolumn{2}{c}{$\begin{array}{c}\text { Viabilidade da fase ninfal } \\
(\%)\end{array}$} \\
\hline 10 & 13 & $15,4 \pm 0,83 \mathrm{c}$ & 46,7 \\
20 & 22 & $11,2 \pm 0,21 \mathrm{a}$ & 67,6 \\
30 & 22 & $12,3 \pm 0,21 \mathrm{a}$ & 76,2 \\
40 & 27 & $13,2 \pm 0,38 \mathrm{~b}$ & 82,3 \\
50 & 27 & $11,8 \pm 0,38 \mathrm{a}$ & 77,8 \\
60 & 20 & $13,3 \pm 0,44 \mathrm{~b}$ & 75,5 \\
Médias & & 12,6 & 66,1 \\
\hline
\end{tabular}

Médias acompanhadas de mesma letra nas colunas não diferem entre si, pelo teste de Scott \& Knott . 
de presas proporcionou um maior período de desenvolvimento do predador. Bush et al. (1993) encontraram período de desenvolvimento semelhante (13,6 dias) quando alimentaram ninfas que deram origem a fêmeas de $O$. insidiosus. Assim, mesmo afetando o período de desenvolvimento do predador, as diferentes densidades de $A$. gossypii testadas são suficientes para que $O$. insidiosus complete o desenvolvimento ninfal.

Todos os ínstares de O. insidiosus apresentaram um aumento linear no consumo de ninfas de $A$. gossypii, de acordo com o aumento na densidade das mesmas (Fig. 1 A, B, C, D e E, Tabela 2). Segundo Hassel (1978), nenhum inseto tem apetite ilimitado, dessa forma, o aumento linear no consumo de presas pode ser teórico pois, de acordo com esse autor, sempre existe uma densidade de presas na qual o predador estabiliza o consumo e o ataque permanece constante.
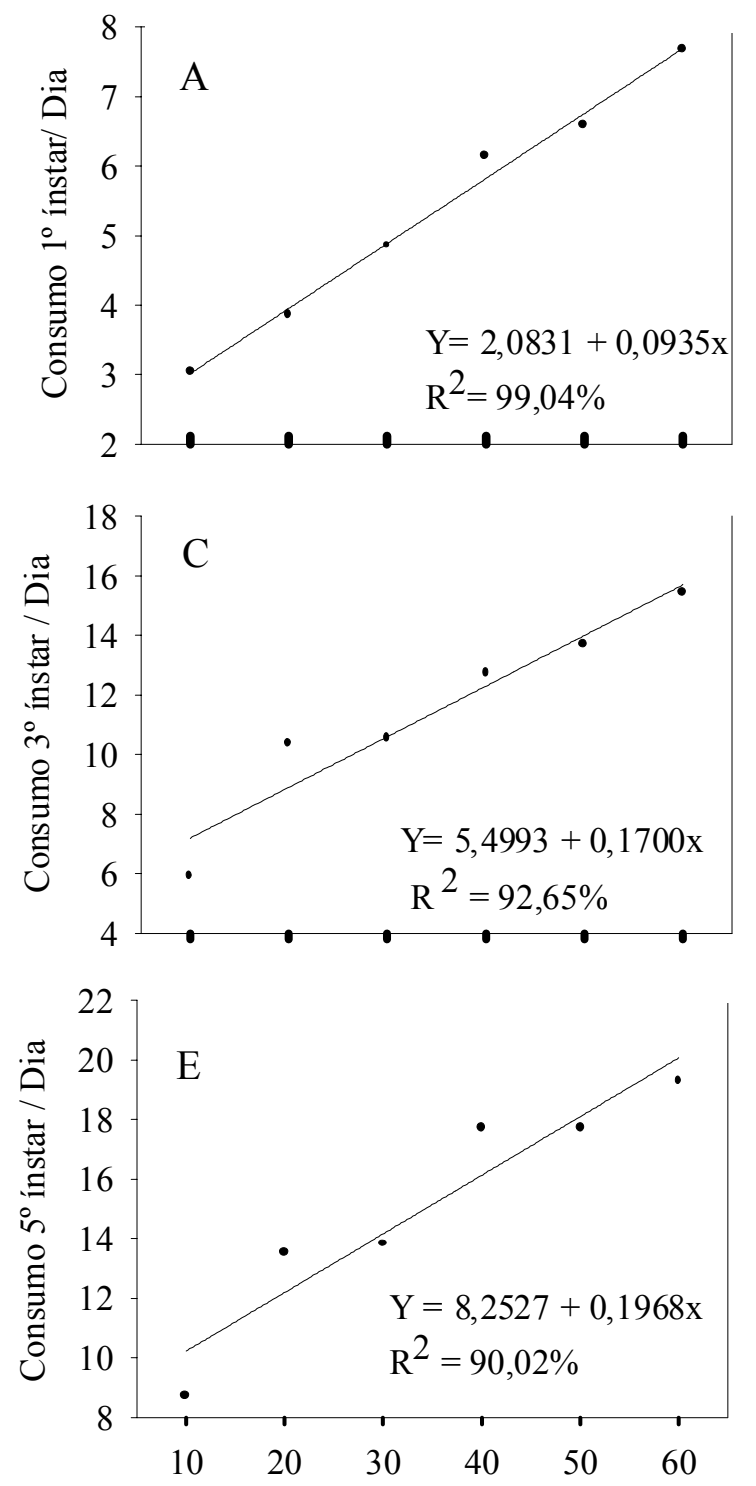

Densidades de A. gossypii
Observou-se a ocorrência de alguns pulgões mortos e parcialmente consumidos, fato que ocorreu principalmente nas densidades mais altas, onde o consumo da presa foi interrompido, algumas vezes, pela movimentação de outros pulgões-presa próximos ao predador. Essas observações concordam com as de Isenhour \& Yeargan (1981a) que verificaram o mesmo comportamento de $O$. insidiosus no consumo do tripes Sericothrips variabilis (Beach, 1896) (Thysanoptera, Thripidae), em maiores densidades de presas. Desta forma, pode se inferir que houve efeito das maiores densidades de presas no comportamento de predação de $O$. insidiosus, ou seja, interrupções da alimentação e aumento no consumo de ninfas de $A$. gossypii. Esse fato é similar ao que ocorre no campo, pois os pulgões formam colônias, nas quais existem grandes densidades de presas em espaços reduzidos.
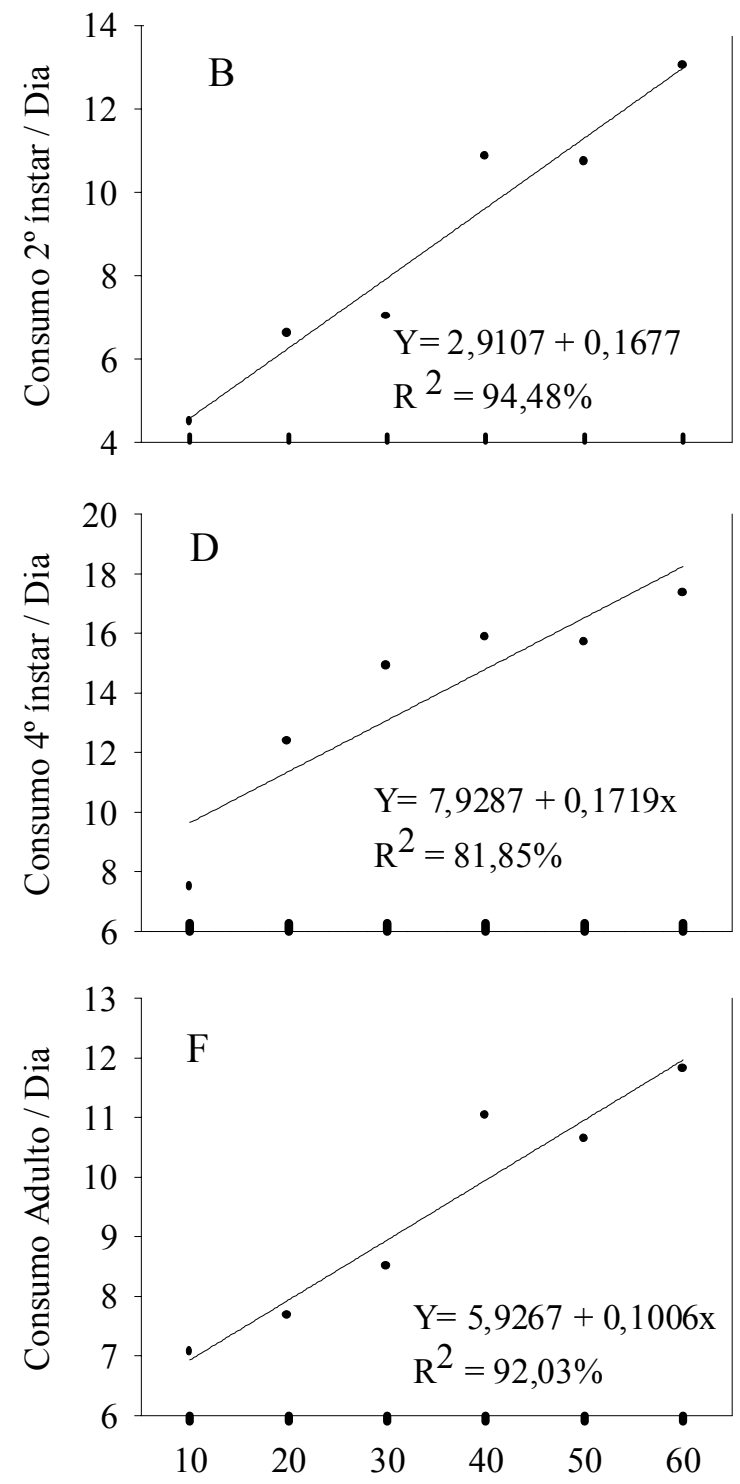

Densidades de A. gossypii

Fig. 1. Consumo de ninfas de Aphis gossypii em diferentes densidades, por ninfas de $1^{\circ}$ (A), $2^{\circ}$ (B), $3^{\circ}$ (C), $4^{\circ}$ (D) $5^{\circ}$ (E) ínstares e adultos (F) de Orius insidiosus, $25 \pm 1{ }^{\circ} \mathrm{C}, 70 \pm 10 \%$ UR e $12 \mathrm{~h}$ de fotofase. 
Ninfas de $1^{\circ}$ ínstar do predador tiveram maiores dificuldades em manusear as presas do que os demais ínstares. O pequeno tamanho (0,50 mm, segundo IsENHOUR \& YEARGAN 1981b) dessas ninfas pode ter contribuído para esse fato, além do comportamento de defesa apresentado pelas ninfas de $A$. gossypii, que se movimentaram ou caminharam na tentativa de afastar o predador.

O consumo de $A$. gossypii por ninfas de $O$. insidiosus (Tabela 2), foi superior àquele encontrado por AlvarAdo et al. (1997) para Orius laevigatus (Fieber, 1860) e Orius majusculus (Reuter, 1879), alimentados com a mesma espécie de pulgão. Esses autores verificaram um consumo médio de 3,9; 7,2 e 4,2 pulgões, para ninfas do $3^{\circ}, 4^{\circ}$ e $5^{\circ}$ ínstares de $O$. laevigatus, respectivamente. A comparação desses resultados com os do presente trabalho reafirma o efeito que a densidade de presas exerce sobre o consumo e a voracidade de $O$. insidiosus em relação às outras espécies, concordando com afirmações de Tommasini \& Nicoli (1993).

A viabilidade de ninfas de $O$. insidiosus variou com as diferentes densidades de presas (Tabela 1), sendo que a densidade de 10 presas/predador/dia proporcionou a menor sobrevivência de ninfas. Em densidades acima dessa, verificouse viabilidade maior que a encontrada por Alvarado et al. (1997) para O. laevigatus alimentado com A. gossypii $(64,3 \%$.) indicando a adeqüabilidade desse pulgão ao desenvolvimento de $O$. insidiosus. Contudo, Bush et al. (1993) relatam que $A$. gossypii não é adequado ao desenvolvimento desse predador, pois a sobrevivência de ninfas é baixa (em torno de $55 \%$ ), o que contraria os resultados obtidos no presente estudo, os quais indicam que a baixa sobrevivência da fase ninfal desse predador alimentado com A. gossypii pode estar relacionada à menor densidade de presas oferecidas e não com a adeqüabilidade da presa ao desenvolvimento desse predador. Outro aspecto, é que esses autores não testaram o efeito da densidade de presas no consumo e desenvolvimento do predador. Assim, é possível que esse predador aumente o seu consumo em densidades maiores no intuito de suprir a possível deficiência nutricional apresentada pela presa, pois maiores densidades de presas propiciaram viabilidade ninfal do predador acima de 75\% (Tabela 1).

Fase adulta $\mathrm{O}$ consumo de presas por adultos de $O$. insidiosus, apresentou um aumento linear de acordo com o aumento na densidade de presas (Fig. 1F e Tabela 2), concordando com resultados de MEIRACKer (1999), onde o consumo de ovos de Anagasta kuehniella (Zeller, 1987) (Lepidoptera, Pyralidae) por esse predador, também apresentou um crescimento linear.

Não foi verificada diferença significativa na média geral do consumo de presas entre machos e fêmeas de $O$. insidiosus $(\mathrm{F}=0,832 ; \mathrm{P}=0,3662$ e $\mathrm{Gl}$ resíduo $=113)$ nas diferentes densidades, com média de 100,3 pulgões consumidos durante toda a fase adulta.

O período de pré-oviposição não foi afetado pela densidade de presas oferecidas $(\mathrm{F}=0,27 ; \mathrm{P}=0,9239$ e $\mathrm{Gl}$ resíduo=34) sendo, em média, 4,8 dias em todas as densidades. Este período foi semelhante ao encontrado por Bush et al. (1993), os quais verificaram que fêmeas desse predador alimentadas com $A$. gossypii apresentaram um período de pré-oviposição de 4,7 dias.

O período de oviposição de $O$. insidiosus foi semelhante para todas as densidades de presa, em média, de 8,9 dias $(\mathrm{F}=$ 1,74; $\mathrm{P}=0,1521$ e Gl resíduo = 34). Bush et al. (1993) observaram um período de oviposição de 5,6 dias para fêmeas alimentadas com A. gossypii, e 4,6 dias para fêmeas alimentadas com Schyzaphis graminum (Rondani, 1852) (Hemiptera, Aphididae). Esta variação pode estar mais relacionada ao tipo de presa do que com a quantidade das mesmas. Mendes et al. (2002) encontraram períodos de pré-oviposição e de oviposição de 3,3 e 44,4 dias respectivamente, quando $O$. insidiosus foi alimentado com ovos de $A$. kuehniella.

A porcentagem de fêmeas que ovipositaram variou com a densidade de presas (Tabela 3). De acordo com GARCIA (1991),

Tabela 2. Consumo médio de ninfas de A. gossypii ( \pm EP) em cada ínstar e na fase adulta de Orius insidiosus em função das diferentes densidades de presa, $25 \pm 1{ }^{\circ} \mathrm{C}, 70 \pm 10 \%$ UR e $12 \mathrm{~h}$ de fotofase.

\begin{tabular}{|c|c|c|c|c|c|c|c|c|}
\hline \multirow{2}{*}{$\begin{array}{c}\text { Densi- } \\
\text { dades }\end{array}$} & \multirow[b]{2}{*}{$\mathbf{N}$} & \multicolumn{7}{|c|}{ Consumo de presas } \\
\hline & & $1^{\circ}$ ínstar & $2^{0}$ ínstar & $3^{\circ}$ ínstar & $4^{0}$ ínstar & $5^{\circ}$ ínstar & $\mathbf{N}$ & Adulto \\
\hline 10 & 13 & $3,0 \pm 0,16 \mathrm{a}$ & $4,5 \pm 0,2 \mathrm{a}$ & $5,9 \pm 0,2 \mathrm{a}$ & $7,5 \pm 0,1 \mathrm{a}$ & $8,8 \pm 0,1 \mathrm{a}$ & 6 & $7,0 \pm 0,30 \mathrm{a}$ \\
\hline 20 & 22 & $3,8 \pm 0,23 \mathrm{a}$ & $6,4 \pm 0,1 \mathrm{a}$ & $10,3 \pm 0,1 \mathrm{~b}$ & $12,3 \pm 0,1 \mathrm{~b}$ & $12,6 \pm 0,1 \mathrm{~b}$ & 11 & $7,8 \pm 0,30 \mathrm{a}$ \\
\hline 30 & 22 & $4,8 \pm 0,26 \mathrm{a}$ & $7,0 \pm 0,1 \mathrm{a}$ & $10,6 \pm 0,2 b$ & $14,6 \pm 0,2 \mathrm{c}$ & $13,8 \pm 0,1 \mathrm{~b}$ & 15 & $8,5 \pm 0,56 b$ \\
\hline 40 & 27 & $6,2 \pm 0,40 \mathrm{~b}$ & $10,8 \pm 0,1 \mathrm{~b}$ & $12,7 \pm 0,0 \mathrm{c}$ & $15,8 \pm 0,1 \mathrm{c}$ & $17,7 \pm 0,2 \mathrm{c}$ & 24 & $11,0 \pm 0,21 \mathrm{c}$ \\
\hline 50 & 27 & $6,6 \pm 0,32 b$ & $10,7 \pm 0,1 \mathrm{~b}$ & $13,7 \pm 0,1 \mathrm{c}$ & $15,7 \pm 0,2 \mathrm{c}$ & $17,7 \pm 0,1 \mathrm{c}$ & 17 & $10,6 \pm 0,39 \mathrm{c}$ \\
\hline 60 & 20 & $7,7 \pm 0,39 \mathrm{c}$ & $13,0 \pm 0,1 \mathrm{~b}$ & $15,4 \pm 0,2 \mathrm{c}$ & $17,4 \pm 0,1 \mathrm{c}$ & $19,3 \pm 0,1 \mathrm{c}$ & 21 & $11,8 \pm 0,47 \mathrm{c}$ \\
\hline \multirow[t]{3}{*}{ Média } & & 5,7 & 9,3 & 12,1 & 14,7 & 16,0 & & 9,8 \\
\hline & & $\mathrm{F}=32,617$ & $\mathrm{~F}=22,608$ & $\mathrm{~F}=318,929$ & $\mathrm{~F}=20,543$ & $\mathrm{~F}=32,917$ & & $\mathrm{~F}=34,513$ \\
\hline & & $\mathrm{P}<0,0001$ & $\mathrm{P}<0,001$ & $\mathrm{P}<0,001$ & $\mathrm{P}<0,001$ & $\mathrm{P}<0,001$ & & $\mathrm{P}<0,001$ \\
\hline
\end{tabular}

Médias acompanhadas de mesma letra nas colunas não diferem entre si, pelo teste de Scott \& Knott . 
Tabela 3. Número total, diário e viabilidade de ovos de Orius insidiosus alimentados com diferentes densidades de ninfas de A. gossypii ( \pm erro padrão), $25 \pm 1{ }^{\circ} \mathrm{C}, 70 \pm 10 \%$ UR e $12 \mathrm{~h}$ de fotofase.

\begin{tabular}{cccccc}
\hline $\begin{array}{c}\text { Densidades de } \\
\text { A. gossypii }\end{array}$ & $\mathrm{N}$ & $\begin{array}{c}\text { \% fêmeas que } \\
\text { ovipositaram }\end{array}$ & $\begin{array}{c}\mathrm{N}^{\mathrm{o}} \text { de ovos } \\
(\mathrm{X} \pm \mathrm{EP})\end{array}$ & $\begin{array}{c}\mathrm{N}^{\mathrm{o}} \text { ovos / dia } \\
(\mathrm{X} \pm \mathrm{EP})\end{array}$ & $\begin{array}{c}\text { Viabilidade } \\
(\%)\end{array}$ \\
\hline 10 & 6 & 16,7 & $2,0 \pm 0,00 \mathrm{a}$ & $1,0 \pm 0,00 \mathrm{a}$ & $0,0 \pm 0,00 \mathrm{a}$ \\
20 & 12 & 50,0 & $11,3 \pm 2,55 \mathrm{a}$ & $1,6 \pm 0,06 \mathrm{a}$ & $52,5 \pm 1,40 \mathrm{~b}$ \\
30 & 10 & 70,0 & $10,7 \pm 1,34 \mathrm{a}$ & $1,3 \pm 0,05 \mathrm{a}$ & $57,9 \pm 0,83 \mathrm{~b}$ \\
40 & 12 & 83,3 & $21,3 \pm 2,35 \mathrm{a}$ & $2,1 \pm 0,09 \mathrm{a}$ & $58,1 \pm 1,49 \mathrm{~b}$ \\
50 & 10 & 90,0 & $17,9 \pm 1,48 \mathrm{a}$ & $1,5 \pm 0,08 \mathrm{~b}$ & $50,1 \pm 0,88 \mathrm{~b}$ \\
60 & 12 & 70,0 & $53,4 \pm 3,049 \mathrm{~b}$ & $3,4 \pm 0,05 \mathrm{c}$ & $72,9 \pm 2,37 \mathrm{c}$ \\
\hline & & $\mathrm{F}=6,01$ & $\mathrm{~F}=12,48$ & $\mathrm{~F}=6,11$ \\
& & $\mathrm{P}=0,0004$ & $\mathrm{P}=0,0001$ & $\mathrm{P}=0,0004$ \\
\hline
\end{tabular}

Médias acompanhadas de mesma letra nas colunas não diferem entre si em teste Scott \& Knott ao nível de 0,01 de probabilidade.

todo animal requer certa quantidade de alimento para sobreviver e uma quantidade maior para crescer e se reproduzir. Assim, fêmeas de $O$. insidiosus submetidas à 10 presas/fêmea, apresentaram o mais baixo percentual de fêmeas que ovipositaram, podendo indicar uma deficiência nutricional proporcionada pela baixa densidade de presas.

O aumento do número de ovos por fêmea de $O$. insidiosus, foi em função do incremento no número de presas. Bush et al. (1993) alimentaram fêmeas de O. insidiosus apenas com A.gossypii e observaram uma média de 19,4 ovos/fêmea. Esses dados são semelhantes aos encontrados no presente trabalho em densidades de até 50 ninfas de $A$. gossypii por indivíduo (Tabela 3), pois na densidade de 60 ninfas, o número de ovos por fêmea do predador foi maior que três vezes o número observado nas demais densidades estudadas. (Fig.2 e Tabela 3).

A viabilidade dos ovos também variou com as diferentes densidades a que foram submetidas as fêmeas desse antocorídeo, sendo que na de 60 presas, houve uma maior viabilidade do que nas demais densidades (Tabela 3 ).

Assim, sob a densidade de 10 presas/predador/dia, $O$. insidiosus apresentou pior desempenho reprodutivo, pois nessa densidade uma menor porcentagem de fêmeas ovipositaram, o número de ovos por fêmea foi baixo e não houve eclosão de ninfas (Tabela 3). Com base nesses resultados, a afirmativa de que predadores do gênero Orius são capazes de se manterem em ambientes de baixa disponibilidade de presas (COLI \& IZRAYLEVICH 1997) deve ser cuidadosamente avaliada, pois baixas densidades de presas podem trazer reflexos na reprodução desses antocorídeos e, conseqüentemente, no estabelecimento desse predador em determinados cultivos, como agente de controle biológico.

Não houve diferença significativa na longevidade de machos e fêmeas de $O$. insidiosus $(\mathrm{F}=0,560, \mathrm{P}=0,4558 \mathrm{e}$ Gl do resíduo $=113)$, bem como nas diferentes densidades $(\mathrm{F}=1,315$, $\mathrm{P}=0,2631$ e Gl do resíduo = 109) que foi, em média, de 9,86 dias. Resultados semelhantes foram obtidos por Bush et al. (1993), verificando que a longevidade de $O$. insidiosus alimentado com A. gossypii foi de 9,5 dias para fêmeas e 6,0 dias para os machos. Entretanto, essa longevidade pode ser considerada baixa quando comparada à longevidade proporcionada por outras dietas, como adultos de $F$. occidentalis (17,1 dias) e ovos de $A$. kuehniella (42 dias) (Tommasin \& Nicoli 1993).

Dessa forma, em programas de controle biológico nos cultivos de crisântemo, pepino, pimentão e outros, onde tripes e pulgões ocorram concomitantemente, deve ser levada em consideração a capacidade crescente de predação de $O$. insidiosus frente ao aumento da densidade de A. gossypii. Esta característica pode facilitar a manutenção e estabelecimento do predador na cultura, podendo ser importante componente de agroecossistemas ajudando a manter várias pragas em níveis abaixo de dano econômico, mesmo sendo uma espécie considerada generalista quanto à predação.

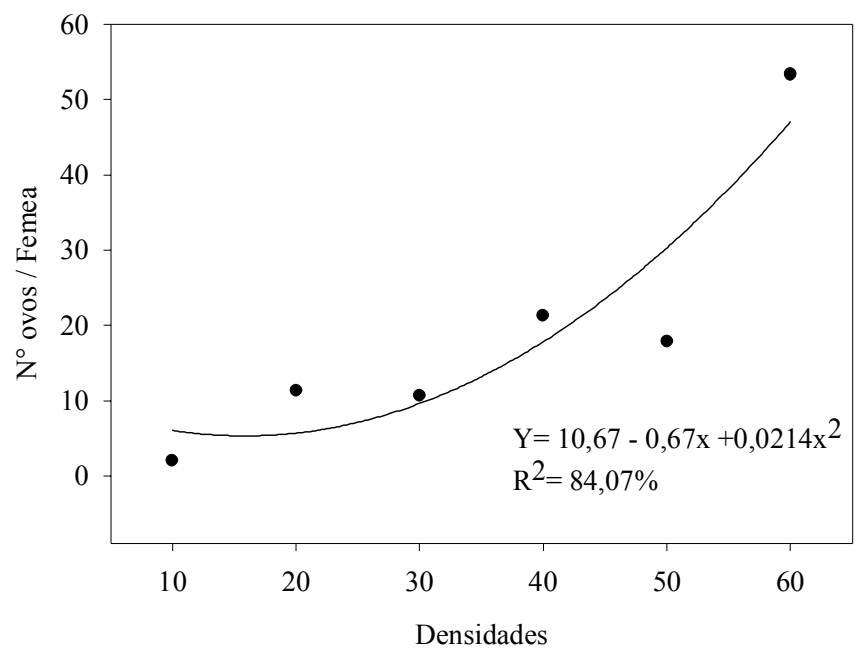

Fig. 2. Número de ovos por fêmeas de $O$. insidiosus alimentadas com diferentes densidades de ninfas de Aphis gossypii, $25 \pm 1{ }^{\circ} \mathrm{C}, 70 \pm 10 \%$ UR e $12 \mathrm{~h}$ de fotofase. 
Agradecimentos. Os autores agradecem à Dra. M. G. Tommasini pela identificação do antocorídeo $O$. insidiosus. À CAPES e ao CNPq pelas concessões das bolsas de estudos e à FAPEMIG pelo suporte financeiro à execução do trabalho .

\section{REFERÊNCIAS}

Albajes, R. \& O. Alomar. 1999. Current and potential use of polyphagous predators, p. 265-275. In: R. Albajes; M. L. Gullino; J. C. Van Lenteren \& Y. Elad (eds.) Integrated Pest and Disease Management in Greenhouse Crops. Dordrecht, Kluwer Academic Publisher, $568 \mathrm{p}$.

Alvarado, P; O. Balta \& O. Alomar. 1997. Efficiency of four heteroptera as predators of Aphis gossypii and Macrosiphum euphorbiae (Hom.: Aphididae). Entomophaga 42: 215-226.

BRioso, P. S. T. 1996. Doenças causadas por vírus em pimentão. Informe Agropecuário 18(184): 74-80.

Bush, L.; T. J. KRING, \& J. R. Ruberson. 1993. Suitability of greenbugs, cotton aphids, and Heliothis virescens eggs for development and reproduction of Orius insidiosus. Entomologia Experimentalis et Applicatta 67: 217-222.

Coll, M. \& S. Izraylevich. 1997. When predator also feed plants: Effect of competition and plant quality on omnivore-prey population dynamics. Annals of the Entomological Society of America 90: $155-161$.

Garcia, M. A. 1991. Ecologia nutricional de parasitóides e predadores terrestres, p. 289-311. In: A. R Panizzi \& J. R. P. PARra (eds.) Ecologia nutricional de insetos e suas implicações no manejo de pragas. São Paulo, Manole, $359 \mathrm{p}$.

Hassel, M. P. 1978. The dynamics of arthropod predator-prey systems. Princeton, Princeton University, $131 \mathrm{p}$.

Imenes S. De L. \& M. A. V. Alexandre (Coord.). 1996. Aspectos fitossanitários do crisântemo. São Paulo, Boletim técnico, Instituto Biológico, $41 \mathrm{p}$.

Isenhour, D. J. \& K. V. YeARgan. 1981a . Interactive behavior of Orius insidiosus (Hem.; Anthocoridae) and Sericothrips variabilis (Thys.: Thripidae): predator searching strategies and prey escape tactics. Entomophaga 26: 213-220.

Isenhour, D. J. \& K. V. Yeargan. 1981b. Effect of temperature on the development of $O$. insidiosus, with note of laboratory rearing. Annals of the Entomological Society of America 74(1): 114116.

Letourneau, D. K. \& M. A. Altieri. 1983. Abundance patterns of a predator, Orius tristicolor (Hemiptera: Anthocoridae), and its prey, Frankliniella occidentalis (Thysanoptera: Thripidae): habitat attraction in polycultures versus monocultures. Environmental Entomology 12(5): 1464-1469.

Malais, M. P. \& W. J. Ravensberg. 1992. The biology of glasshouse pests and their natural enemies. Netherlands, Roddernris: Koppert, $109 \mathrm{p}$.

Meiracker, A. F. van Den. 1999. Biocontrol of western thrips by heteroteran bugs. PhD Thesis. Amsterdam, University of Amterdam, $147 \mathrm{p}$.

Mendes, S. M. \& V. H. P. Bueno. 2001. Biologia de Orius insidiosus (Say) (Hemiptera: Anthocoridae) alimentado com Caliothrips phaseoli (Hood) (Thysanoptera: Thripidae). Neotropical Entomology 30 (3): 423-428.

Mendes S. M.; V. H. P. Bueno; V. M. Argolo \& L. C. P. Silveira. 2002. Type of prey influences biology and consumption rate of Orius insidiosus (Say) (Hemiptera, Anthocoridae). Revista Brasileira de Entomologia 46(1): 99-103.

Sсотт, А. J. \& M. A. Клотт. 1974. A cluster analyses method for grouping means in the analyses of variance. Biometrics 30: 507-512.

Tommasini, M. G. \& G. Nicoli. 1993. Adult activity of four Orius species reared on two preys. Bulletin IOBC/WPRS 16: 281-184.

VAN Schelt, J. 1993. Market-driven research and development in biological control. Pesticide Science 37: 405-409. 